\title{
Emission of light through thin silver films via near-field coupling to surface plasmon polaritons
}

\author{
G. Winter ${ }^{\mathrm{a})}$ and W. L. Barnes \\ Electromagnetic Materials Group, University of Exeter, Exeter EX4 4QL, United Kingdom, EU
}

(Received 27 June 2005; accepted 9 December 2005; published online 1 February 2006)

\begin{abstract}
We show that the emission of light from a dye layer through an adjacent thin silver film is maximal for a silver thickness of approximately $50 \mathrm{~nm}$. This effect is explained as the result of competition between enhancement of the electric field at the metal surface due to the excitation of a surface plasmon-polariton mode, the amount of power coupled to the surface plasmon-polariton mode, and the attenuation of the field transmitted through the silver, all three of which vary with metal thickness. We indicate how these findings may be of relevance in the design of some surface plasmon-polariton-based fluorescence biosensing schemes. () 2006 American Institute of Physics.
\end{abstract}

[DOI: $10.1063 / 1.2170426$ ]

The emission of light through thin metal films is of interest from a variety of applied perspectives, including topemitting organic light-emitting diodes ${ }^{1}$ and fluorescence biosensors using surface plasmon polaritons (SPPs). ${ }^{2}$ Thin silver films are also of topical interest for their possible use as lenses capable of beating the usual diffraction limit. ${ }^{3,4}$ In both lensing and SPP mediated emission of light, there appears to be an optimum metal film thickness. Recently, the SPP mediated transmission of light through a thin silver film using a scheme in which the SPP modes were initially excited via surface-roughness coupling was investigated. ${ }^{5,6}$ More recently the same phenomenon was explored using the more controlled form of coupling to SPPs offered by wavelength-scale periodic gratings. ${ }^{7}$ In these studies, a silver film thickness of $\sim 50 \mathrm{~nm}$ was found to optimize the SPP mediated transmission of incident plane-wave light through the metal. Here, we explore a closely related phenomenon, SPP mediated emission of light through a thin metal film, i.e., the transmission of the near-field emission of light from molecules through a metal film. One might expect, based on the work on the transmission of incident plane-wave light, that a similar optimum film thickness would exist for SPP mediated emission through a metal film. However, the emission process is complicated by the presence of the metal film since this film alters the decay properties of the emitting molecules. ${ }^{8}$ Here, we specifically addressed the thickness dependence of SPP mediated emission through a thin silver film to explore this phenomenon further.

We followed a well established technique that uses emissive dye molecules to excite the SPP modes of a thin metal film that was deposited on the base of a prism. ${ }^{9-12}$ Dye molecules may excite SPP modes on the dye/silver interface, and these SPP modes may in turn decay to produce light in the prism. Using this technique, we measured how the strength of the SPP mediated emission into the prism varied with silver film thickness. This technique has recently been called surface plasmon coupled emission ${ }^{2}$ and is a reversal of the usual Kretschmann-Raether attenuated total reflection approach for the excitation of SPPs. ${ }^{13}$ In fact, this type of structure (Fig. 1) supports two SPP modes, one associated with the dye-silver interface, the other associated with the silver-

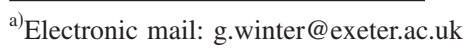

silica interface. Only the SPP associated with the dye-silver interface can couple to light in the prism, and it is this mode we investigate here. The SPP associated with the silver-silica interface has too much momentum to couple to light in the prism and any power coupled from the dye to this mode is eventually lost as heat, we refer to this below as the lossy SPP mode.

A diagram of our experimental arrangement is shown in Fig. 1. The samples consisted of a silica substrate onto which a layer of silver, and then a layer of the dye aluminium tris-(8-hydroxyquinoline) were evaporated. Before adding the dye layer, the thickness and relative permittivity of the silver were determined by fitting theoretical models to angle dependent reflectivity data. This procedure was repeated after the dye was added, thereby providing the thickness and relative permittivity of the $\mathrm{Alq}_{3}$.

To explore the SPP mediated emission of light, the samples were index matched onto the base of a silica prism and the dye molecules were optically excited with $410 \mathrm{~nm}$ light from a diode laser. SPP mediated emission through the silver into the prism was collected with a lens and passed to a fiber-coupled spectrometer, spectra were recorded using a charge coupled device for a range of emission angles.

Figure 2 shows four experimental spectra, all obtained at the same polar emission angle. The spectra from the samples containing no silver (i.e., dye deposited directly onto silica)

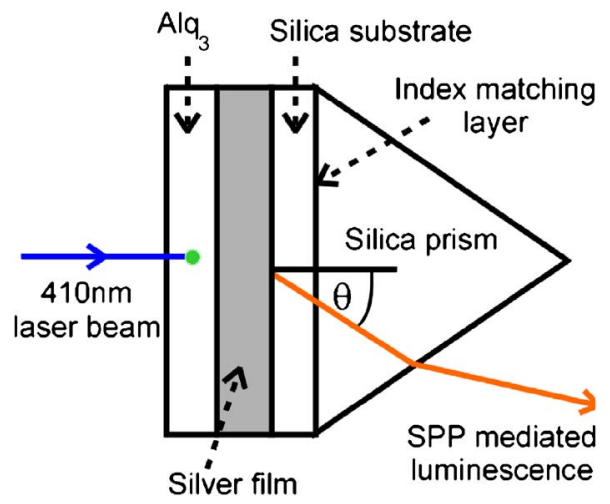

FIG. 1. (Color online) Schematic of experimental system. The silica substrate supports a silver film $18-91 \mathrm{~nm}$ thick. $30 \mathrm{~nm}$ of $\mathrm{Alq}_{3}$ is evaporated on top of the silver and the substrate is index matched to a silica prism. 


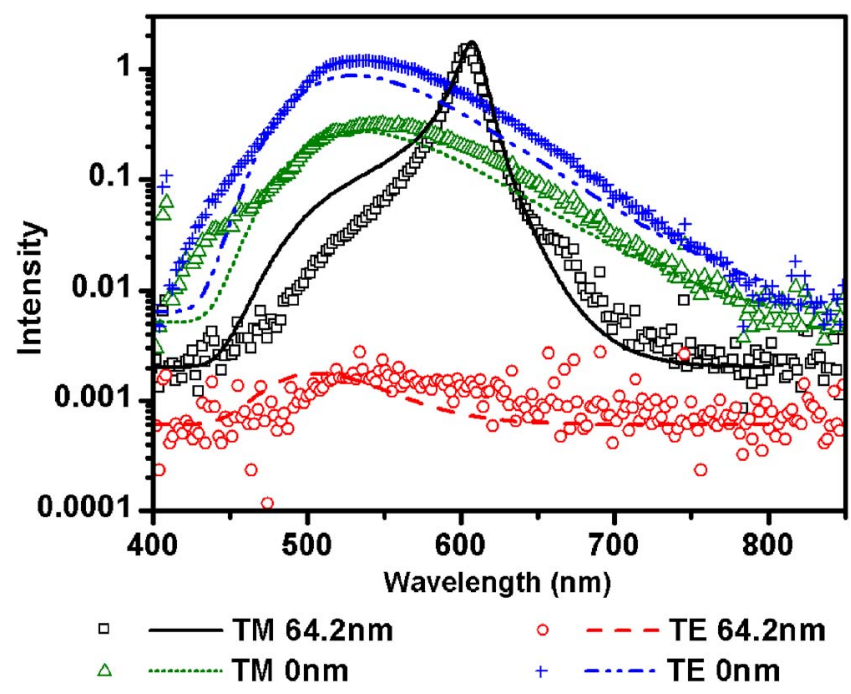

FIG. 2. (Color online) (Density of data points reduced for clarity.) Experimental and theoretical TM and TE polarized luminescence spectra collected through $64.2 \mathrm{~nm}$ silver, and emission spectrum of dye through no silver. All spectra displayed were collected or simulated at a polar emission angle of $\theta=63.4^{\circ}$.

for both transverse electric (TE) and transverse magnetic (TM) polarizations show the expected characteristic emission spectrum of $\mathrm{Alq}_{3}$. The addition of a $64.2 \mathrm{~nm}$ thick silver film leads to a significant decrease in both the TE and TM polarized emission, except for the striking appearance of a new peak in the TM-polarized photoluminence. This peak arises from SPP mediated emission and remarkably is seen to be stronger (around the peak) than the emission in the absence of the metal. Theoretical simulations using a classical dipole antenna model ${ }^{14}$ are indicated in Fig. 2 by lines and show reasonable agreement with the experimental data.

To confirm that the TM polarized peaks arise from SPP mediated emission, the PL spectra at specific polar emission angles were compared with wavelength-dependent reflectivity data acquired at the same angles. These data showed that the wavelength of the PL emission peak corresponds very well with the wavelength of the reflectivity minimum, the position of which followed the expected dispersion from the SPP mode supported by this structure. ${ }^{15}$

Spectra similar to Fig. 2 were acquired for a wide range of emission angles. From these data, the TM polarized emission as a function of silver film thickness could be deduced, and is shown in Fig. 3. Since the angle that offers best coupling between light and the SPP is a function of silver film thickness, data for each silver film thickness were chosen from the angle that gave the strongest signal. There is clearly a peak in the transmission, occurring when the silver film is $\sim 50 \mathrm{~nm}$ thick. The theoretical simulation, again based on the classical radiating dipole model, calculated for the wavelength of $625 \mathrm{~nm}$, shows good agreement with the experimental data.

This peak in the emitted light as a function of silver film thickness arises because of a competition between the enhancement of the electric field ${ }^{16}$ at the silver-dye interface and attenuation through the metal film. The increasing electric field enhancement arises from the decrease in the radiative decay rate of the SPP as the silver film thickness increases. In turn, the enhanced field strength leads to a greater probability of the dye molecules coupling to the SPP mode, owing to the associated increase in the density of optical

Downloaded 25 Jul 2008 to 144.173.6.22. Redistribution subject

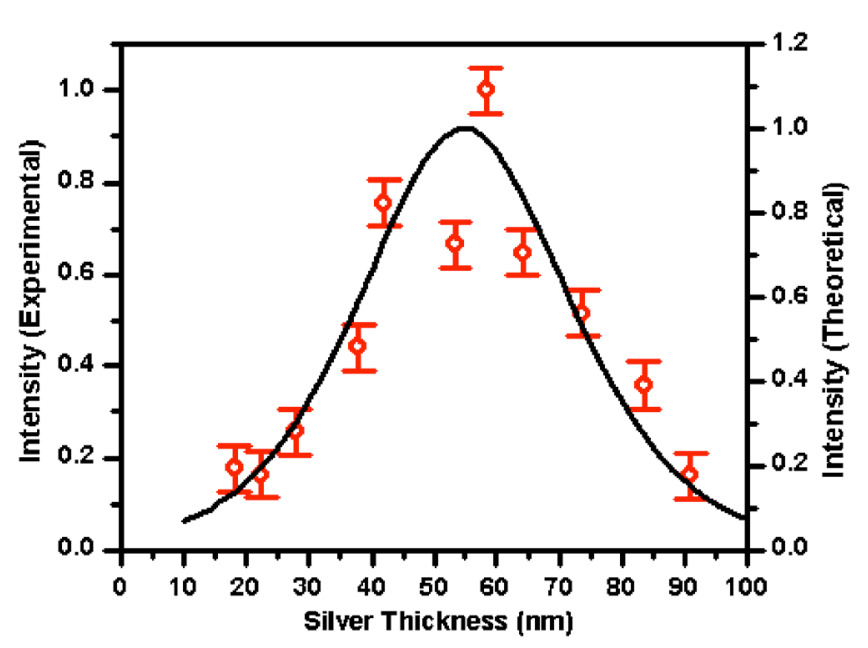

FIG. 3. (Color online) Experimental and theoretical maximum emission through silver as a function of silver thickness. Data and simulation were obtained at a wavelength of $625 \mathrm{~nm}$, with the simulation using a silver permittivity

$\varepsilon=-17.8+0.49 i$.

states. ${ }^{8}$ The increasing probability of coupling to SPPs is in competition with the rising levels of attenuation as the silver film is made thicker. For very thin silver films $(20-50 \mathrm{~nm}$ thick), the increasing field enhancement is the stronger effect, above $50 \mathrm{~nm}$ attenuation dominates.

To explore one possible consequence of these results, we undertook simulations ${ }^{13}$ of the power transmitted through the silver film from a system more suited to biomolecular sensing applications. For this simulation, isotropically aligned emissive dye molecules were positioned $5 \mathrm{~nm}, 15 \mathrm{~nm}$, and $30 \mathrm{~nm}$ from the planar silver surface, within a semi-infinite layer of water. The silver separates the water from a semiinfinite layer of silica. Both the silver and silica are assumed to have similar properties to the materials used in the experiments described above $\left(\varepsilon_{\mathrm{Ag}}=-16+0.44 i, \varepsilon_{\text {Silica }}=2.126\right.$ at a wavelength of $600 \mathrm{~nm}$ ).

Figure 4 shows the fraction of the total power emitted from the dye that is transmitted radiatively into the water and

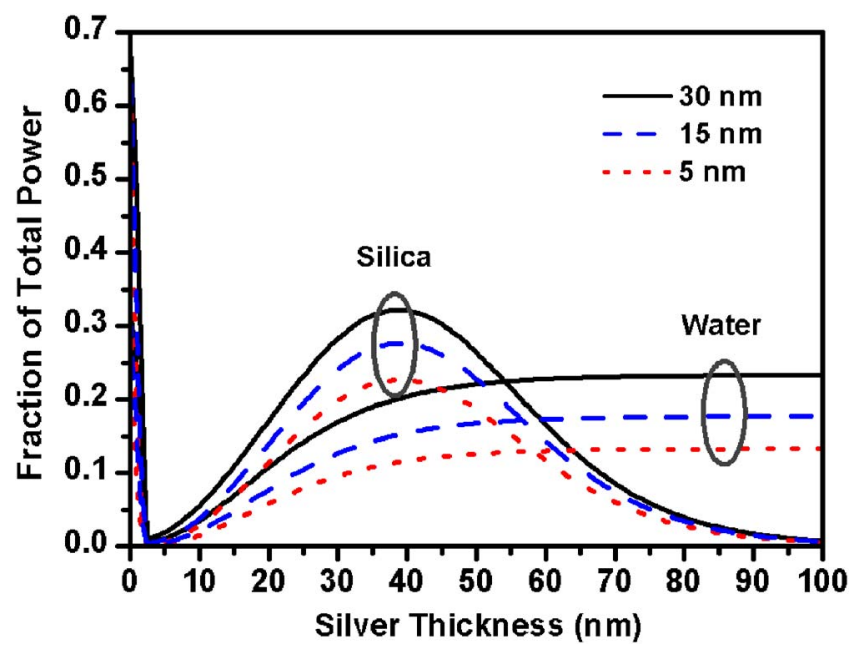

FIG. 4. (Color online) The fraction of the total power emitted from the dye that is transmitted radiatively into the silica and the water. The emissive dipole is situated $5 \mathrm{~nm}, 15 \mathrm{~nm}$, or $30 \mathrm{~nm}$ from the silver within a semiinfinite layer of water, and the silver has a permittivity of $\varepsilon=-16+0.44 i$ at a wavelength of $600 \mathrm{~nm}$. The quantum efficiency of the emitter was taken to be unity. 
into the silica. The power radiated into the water gradually increases and then saturates as the silver thickness is increased from $10 \mathrm{~nm}$ to $100 \mathrm{~nm}$. The power radiated into the silica varies markedly with the silver thickness and peaks when the silver is approximately $50 \mathrm{~nm}$ thick, before decaying in an exponential manner. There is a marked reduction in power radiated into both the water and the silica for silver films $\sim 4 \mathrm{~nm}$ thick. This arises because as the thickness of the silver film is reduced, the fraction of power coupled to the lossy SPP associated with the silver-silica interface increases to such an extent that it is the dominant emission pathway and leaves very little power radiated into either water or silica. As noted above, this mode cannot couple to light in the prism; all power coupled to this mode is dissipated within the silver.

In the absence of any silver film, $\sim 70 \%$ of the power emitted by the dye goes into the silica, the remaining $\sim 30 \%$ into the water. The higher fraction in the silica is due to the higher refractive index of silica compared to water, thus giving a greater density of optical states in the silica. ${ }^{8}$ Figure 4 shows that with a silver film present and a dye-silver spacing of $30 \mathrm{~nm}$, the power emitted into the silica through $40 \mathrm{~nm}$ of silver can be up to $30 \%$ of the total emission from the dye. Although this is less than the fraction of power that goes into the silica in the absence of the metal film, the use of a metal film allows surface chemistry to be used for the moleculespecific binding required for biosensing. Further, only emission originating close to the metal film contributes to SPP mediated emission, thus helping exclude background fluorescence.
In summary, we have shown that the conditions leading to an optimum metal thickness for SPP mediated transmission of light through a metal film also occur for SPP mediated emission of light through a metal film. In both cases, this optimum thickness is approximately $50 \mathrm{~nm}$. These results have relevance for a variety of applications, including the development of fluorescence biosensors and top-emitting organic light-emitting diodes.

The authors would like to acknowledge financial support from the Surface Plasmon Photonics Project (NMP-CT2003-505699) and the Royal Society, and would like to thank Dr. I. R. Hooper for many productive discussions and Qinetiq for assisting with sample fabrication.

${ }^{1}$ M.-H. Lu, M. S. Weaver, T. X. Zhou, M. Rothman, R. C. Kwong, M. Hack, and J. J. Brown, Appl. Phys. Lett. 81, 3921 (2002).

${ }^{2}$ J. R. Lakowicz, Anal. Biochem. 324, 153 (2004).

${ }^{3}$ J. B. Pendry, Phys. Rev. Lett. 85, 3966 (2000).

${ }^{4}$ N. Fang, H. Lee, C. Sun, and X. Zhang, Science 308, 534 (2005).

${ }^{5}$ N. Fang, Z. W. Liu, T. J. Yen, and X. Zhang, Opt. Express 11, 682 (2003).

${ }^{6}$ Z. Liu, N. Fang, T. Yen, and X. Zhang, Appl. Phys. Lett. 83, 5184 (2003).

${ }^{7}$ A. Giannattasio, I. R. Hooper, and W. L. Barnes, Opt. Express 12, 5881 (2004).

${ }^{8}$ G. W. Ford and W. H. Weber, Phys. Rep. 113, 195 (1984).

${ }^{9}$ I. Pockrand, A. Brillante, and D. Möbius, Chem. Phys. Lett. 69, 499 (1980).

${ }^{10}$ W. H. Weber and C. F. Eagen, Opt. Lett. 4, 236 (1979).

${ }^{11}$ H. J. Simon and J. K. Guha, Opt. Commun. 18, 391 (1976).

${ }^{12}$ R. E. Benner, R. Dornhaus, and R. K. Chang, Opt. Commun. 30, 145 (1979).

${ }^{13}$ E. Kretschmann and H. Raether, Z. Naturforsch. A 23A, 2135 (1968).

${ }^{14}$ J. A. E. Wasey and W. L. Barnes, J. Mod. Opt. 47, 725 (2000).

${ }^{15}$ H. Raether, Surface Plasmons (Springer, Berlin, 1988).

${ }^{16}$ W. H. Weber and G. W. Ford, Opt. Lett. 6, 122 (1981). 